\title{
In situ measurement of cavity length variation across the transverse section of broad-area vertical-cavity surface-emitting lasers
}

\author{
Stéphane Barland ${ }^{\mathrm{a})}$ and Francesco Marino \\ Institut Mediterrani d'Estudis Avançats, (CSIC-Universitat de les Illes Balears), C/ Miquel Marqués, 21, \\ E-07190 Esporles, Spain \\ Massimo Giudici and Jorge Tredicce \\ Institut Non Linéaire de Nice, 1361 Route des Lucioles, F-06560 Valbonne, France \\ Salvador Balle \\ Institut Mediterrani d'Estudis Avançats, (CSIC-Universitat de les Illes Balears), C/ Miquel Marqués, 21, \\ E-07190 Esporles, Spain
}

(Received 17 December 2002; accepted 7 July 2003)

\begin{abstract}
We present a method to measure the cavity length variations of a broad-area vertical-cavity surface-emitting laser. The method is based on the observation of an inhomogeneous nonlinear response of the system under coherent optical injection. Due to the presence of a cavity resonance frequency gradient in the system, the boundary of a modulational instability manifests itself as a separation of the device in two distinct spatial regions. The observation of the position of this boundary depending on parameters reveals the degree of homogeneity of the sample and allows a direct measurement of the spatial variations of the cavity resonance frequency. (C) 2003 American Institute of Physics. [DOI: 10.1063/1.1608463]
\end{abstract}

Among the coherent light sources commonly used in telecommunications, vertical-cavity surface-emitting lasers (VCSELs) are of growing interest due to their considerable improvements in terms of threshold current as well as available coherent optical power. In fact, they are now considered powerful enough for either serving as pump-sources in optical fiber-amplifier systems ${ }^{1}$ or to act by themselves as optical amplifiers. $^{2,3}$

The vertical cavity geometry allows for intrinsic singlelongitudinal mode behavior, easy coupling of the device to fiber, and high device density on the wafer. During the epitaxial growth process, some degree of (intentional or not) radial variation in the thickness of the different bulk layers defining the laser structure is introduced, with the result that individual VCSELs on the same wafer have different tuning conditions between gain peak and cavity resonance frequency. 4

Among the applications of VCSELs, a particularly appealing one is the use of broad area devices in an amplifier configuration which has been suggested ${ }^{5,6}$ for applications in parallel data processing. Such an application is based on the generation of transverse structures which originate from the nonlinearity of the matter-field interaction in the device and are not determined by the transverse boundary conditions. From this point of view, the uniformity of the device is a very important issue, and the nonuniformity introduced in the epitaxial growth could then be a highly limiting factor: since the cavity length varies along the chip, it is also expected to vary in a single device, specially if broad area.

So far, estimations of the changes in cavity length along a broad area device are indirectly obtained by measuring the wavelength at threshold of several, small diameter VCSELs

${ }^{a)}$ Electronic mail: stephane@ino.it placed on different points of the wafer. From here it is possible to calculate the value and direction of the gradient of the wafer thickness and, therefore, the cavity resonance frequency gradient of every device obtained from the wafer. This method is thus based on measurements of several devices in the same wafer together with the information of their respective positions on the wafer, which is only available to the manufacturer. In addition, it must be noticed that if the temperature varies from one VCSEL to the other, the measurement will be contaminated by the different amount of thermal shift that the cavity mode will experience. This effect is unavoidable in the present situation, because-due to the different tuning condition-different devices have different threshold currents that lead to different amounts of selfheating. Assessing the magnitude of this contribution seems rather involved.

We demonstrate here a way of precisely and directly measuring the spatial variations of the cavity resonance frequency in a single broad area device, whitout requiring any previous information either about value or direction of the possibly existing gradient. The method is based on the injection of a uniform and coherent beam into the broad area VCSEL to be tested, which is maintained below threshold and therefore acts as a broad-area VCSEL semiconductor optical amplifier (BA-VCSOA). The theoretical foundation of our method for the determination of the spatial variations of the cavity resonance frequency is nonlinear pattern formation in high Fresnel number systems. ${ }^{7,8}$ Theoretical studies of homogeneous, high Fresnel number BA-VCSOAs ${ }^{5,6}$ have shown that the amplified light may exhibit a uniform or nonuniform profile depending on the current injected into the BA-VCSOA and the intensity and optical frequency of the injection beam. These studies have shown that, for fixed current and optical power injected into the device, there is a range of detuning values between the longitudinal resonance 


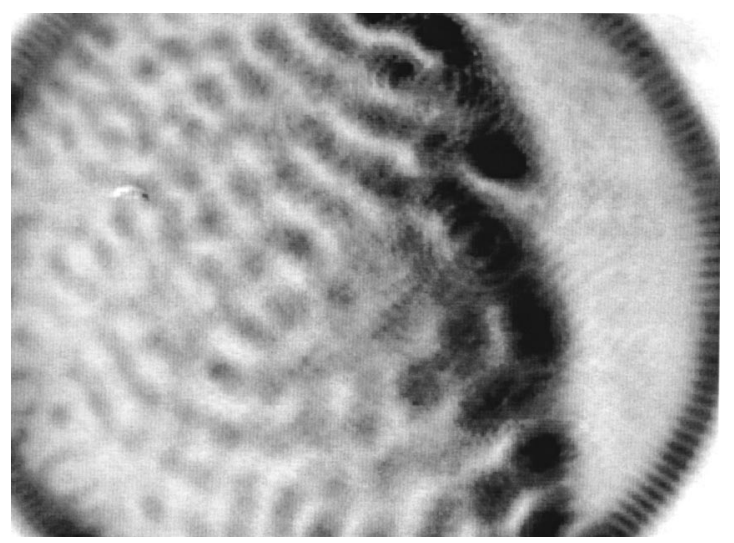

FIG. 1. Typical near field emitted by the VCSEL below threshold under the injection of a uniform coherent field of $8 \mathrm{~mW}$ power. By convention, high reflected intensity is dark and low reflected intensity is white.

of the cavity and the frequency of the injected optical field where the uniform state is unstable and gives rise to an optical pattern through a so called "modulational instability" (MI). ${ }^{9-11}$ The transverse wavelength of this nonuniform spatial structure that spans the whole system depends on the detuning between the cavity longitudinal resonance and the injection optical frequency since this parameter determines the most unstable wave vector at the instability threshold (for a detailed discussion see, e.g., Ref. 12). Therefore, the existence of the MI then defines in parameter space a region where structures are formed and another one where the uniform solution is stable. In a system where parameters depend on space across the transverse section, we will demonstrate that the separation between the two regions in terms of parameters manifests as a separation between two regions in geometrical space. One can then associate the spatial variations of the output of the system with the spatial inhomogeneity of the parameters.

The VCSEL under study is an oxidized bottom emitter ${ }^{1}$ with Bragg mirrors consisting of 20.5 pairs on the bottom side and 30 pairs on the top side, and three quantum wells emitting around $970 \mathrm{~nm}$ close to threshold. Its diameter is $150 \mu \mathrm{m}$. An external-cavity laser in Littmann configuration provides the injection beam, which can be tuned around the emission frequency of the VCSEL at threshold. After passing through an optical isolator (Gsänger model FR 500/1100, return loss $>30 \mathrm{~dB}$ ) to avoid any disturbance due to reflections from the BA-VCSOA, the beam of the injection laser is prepared such that its intensity is almost uniform across the whole input mirror of the amplifier, and injected into it along the optical axis of the BA-VCSOA. The intensity of the injection beam is of the order of a few milliwatts but even weaker intensities can be used, as long as this value is kept constant in time and uniform in the input plane. The reflected output of the amplifier is monitored by a charge coupled device camera, on which we form an image of the emitted near field. Since the VCSEL is used as an amplifier, no dynamics occurs, ${ }^{5,6}$ and therefore no fast detection is required.

A typical output is shown in Fig. 1. As expected in such a broad-area device, a transverse structure forms. The output clearly shows a left part where a structure is formed, and a right part where the reflected intensity is uniform at a low Downloaded 16 Sep 2003 to 130.206.32.193. Redistribution subject

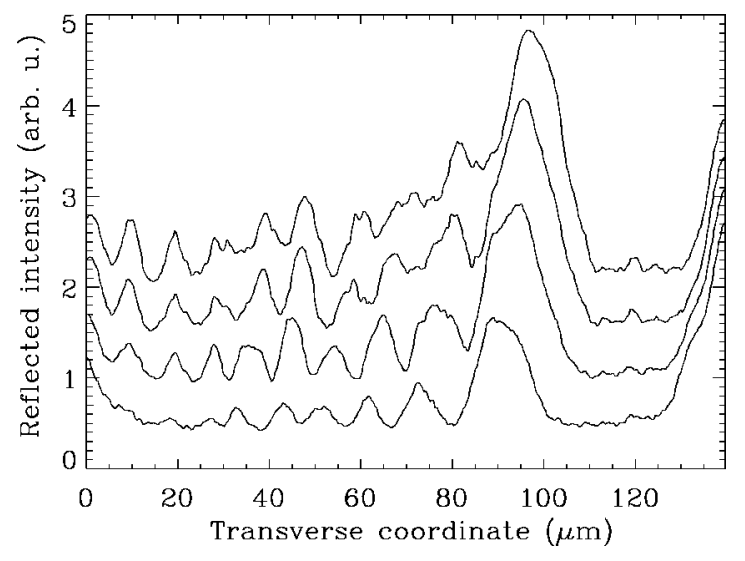

FIG. 2. Cut along the horizontal diameter of the near field image for different injection powers (from 1 to $8 \mathrm{~mW}$ from bottom to top. The different curves have been offset for clarity.

value, these two zones being separated by a region of maximal amplification. Moreover, the pitch of the spatial structure uniformly decreases to the left of the device. We have here to emphasize that this asymmetry is not due to misalignment of the injected beam: the axis of the injected beam is parallel to the emission axis of the VCSEL, and the injected beam is practically uniform over the input plane. Furthermore, even if one deliberately misaligns the injection beam, this asymmetry survives: although the patterns present on the left side of the device are perturbed, the right side does not show the formation of any kind of structure. Finally, the maximum present on the rightmost part of the picture also exists without the injection beam and is due to a higher level of spontaneous emission in this particular region, because of current crowding close to the insulating boundaries. The observation of the spatial position of the boundary between the two domains (with and without spatial structure) depending on parameters is at the core of the scheme that we propose.

The nature of this area of maximal amplification can be understood by recognizing that it constitutes the boundary between a spatial region where the system shows spontaneous pattern formation and the spatial region where the output is uniform. We recall that in a homogeneous system, for all other parameters fixed, this MI boundary is defined in $p a$ rameter space only by a certain critical detuning $\theta_{c}$ between the injected field frequency and the longitudinal cavity resonance: for detunings larger than $\theta_{c}$ a pattern develops and viceversa. Hence, in a system where the cavity resonance varies in real space along one direction the MI boundary then manifests itself as a separation between a spatial region where transverse structures form (in the spatial region where parameters are such that the uniform solution is unstable), and a uniform one (in the spatial region where parameters are such that the uniform solution is stable). It therefore maps into real space the MI region in parameter space due, in our case, to the non-parallel mirrors forming the VCSEL cavity.

This interpretation is further supported by the observation that the position of the boundary depends on the intensity of the injected field, as shown in Fig. 2: indeed, numerical and analytical studies of the homogeneous system ${ }^{5,6}$ show that $\theta_{c}$ depends on the intensity of the injected field, hence, in inhomogeneous systems the spatial variations of the cavity resonance frequency should lead to a displacement to AlP license or copyright, see http://ojps.aip.org/aplo/aplcr.jsp 


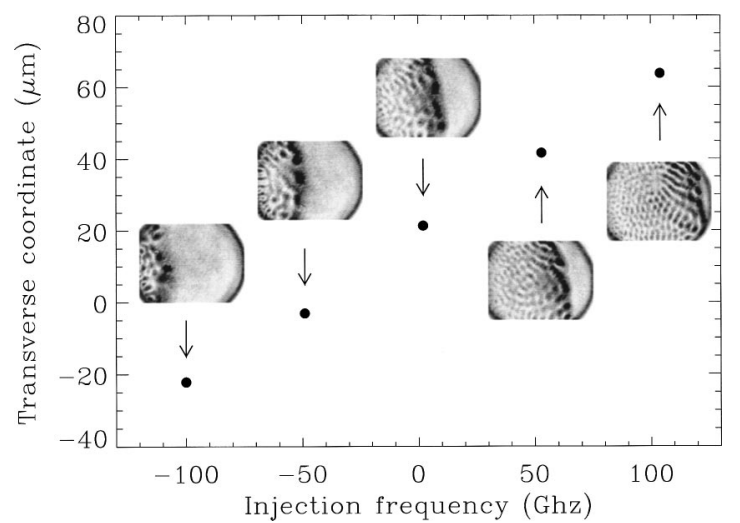

FIG. 3. Abscissa of the point of space where $\omega_{c}-\omega_{i}=\theta_{c}$ as a function of the optical injection frequency $\omega_{i}$ with respect to an arbitrary origin.

of the MI boundary in real space. Finally, note that in a homogeneous system, if the intensity of the injected field were kept constant while varying its optical frequency, the pitch of the spatial pattern would change; hence, in an inhomogeneous system, this should produce a rigid shift of the whole pattern by displacing the boundary of the MI to the point where the critical detuning condition is satisfied, in good agreement with our observations (see insets in Fig. 3).

Since $\theta_{c}$ is linear on the frequency of the injected field, this allows tracking of the spatial changes in cavity length of the amplifier. As clearly seen in the insets of Fig. 3, each injected frequency determines a well-defined position for the MI boundary, which allows determination of the variation of the optical length of the resonator along one of its transverse dimensions. By plotting the abscissa of the MI boundary as a function of the injected field frequency $\omega_{i}$, we find a linear variation of the cavity length along the horizontal dimension with a slope of $2.34 \mathrm{GHz} \mu \mathrm{m}^{-1}$, which leads to a resonance frequency difference of $351 \mathrm{GHz}$ between one side and the other of the amplifier. This value is in excellent agreement with the value found from wafer measurements. ${ }^{4}$

Finally, it is worth discussing the practical measurement limits of the method here presented. In the first place, the injected beam has to allow for fine tuning as compared to the total change in cavity resonance frequency in order to track the spatial changes of the MI boundary; thus, a tunable external cavity laser that can be fine tuned through temperature control should suffice in most cases. On the other hand, the resolution achievable with our method is only limited by the spatial extension of the MI boundary and the correlation length of the system. The correlation length depends on the strength of the nonlinear field-matter interaction: in order to observe the behavior described earlier, the injected intensity should not be too weak since this would lead to a broadening of the falling edge of the boundary between the two regions. We have demonstrated here that a power of $1 \mathrm{~mW}$ distributed over a device of $150 \mu \mathrm{m}$ is sufficient for a successful implementation of the method if the VCSEL is close enough to threshold.

In conclusion, we have presented a method to reveal the presence of a cavity resonance frequency inhomogeneity along the transverse dimension(s) of a vertical cavity surface emitting laser. While the presence of such inhomogeneities could so far only be detected by indirect means involving several devices of the same chip, we have demonstrated the possibility of a direct and quantitative measurement of the variations of the cavity resonance frequency along space. Since the method is based on nonlinear effects, some minimal conditions in terms of optical power have to be fulfilled, but we provided the demonstration that low injection power is sufficient for a successful application of the method.

The authors ackowledge financial support from EU through Projects PIANOS (ESPRIT) and VISTA (HC-TMR) and from the spanish government through Project No. TIC99-0645-C05-02.

${ }^{1}$ M. Grabherr, R. Jäger, M. Miller, C. Thalmaier, J. Herlein, and K. J. Ebeling, 10, 1061 (1998).

${ }^{2}$ J. Piprek, S. Björlin, and J. E. Bowers, IEEE J. Quantum Electron. 37, 127 (2001).

${ }^{3}$ S. Björlin and J. E. Bowers, 38, 61 (2002).

${ }^{4}$ M. Grabherr and T. Knödel, PIANOS internal report, Ulm, June 2001.

${ }^{5}$ M. Brambilla, L. A. Lugiato, F. Prati, L. Spinelli, and W. J. Firth, Phys. Rev. Lett. 79, 2042 (1997).

${ }^{6}$ L. Spinelli, G. Tissoni, M. Brambilla, F. Prati, and L. A. Lugiato, Phys. Rev. A 58, 2542 (1998).

${ }^{7}$ S. P. Hegarty, G. Huyet, J. G. McInerney, and K. D. Choquette, Phys. Rev. Lett. 82, 1434 (1999).

${ }^{8}$ W. J. Firth and A. Lord, 7, 255 (1998).

${ }^{9}$ M. C. Cross and P. C. Hohenberg, Rev. Mod. Phys. 65, 851 (1993).

${ }^{10}$ F. T. Arecchi, S. Boccaletti, and P. L. Ramazza, Phys. Rep. 318, 1 (1999).

${ }^{11}$ L. A. Lugiato, M. Brambilla, and A. Gatti, Adv. At., Mol., Opt. Phys. 40, 229 (1999).

${ }^{12}$ W. J. Firth and A. J. Scroggie, Europhys. Lett. 26, 521 (1994). 